\title{
Passive scalar tagging for the study of coherent structures in the plane mixing layer
}

\author{
B. R. Ramaprian \\ Department of Mechanical and Materials Engineering, Washington State University, Pullman, \\ Washington 99164-2920
}

N. D. Sandham, M. G. Mungal, and W. C. Reynolds

Department of Mechanical Engineering, Stanford University, Stanford, California 94305

(Received 17 February 1989; accepted 29 August 1989)

\begin{abstract}
Data obtained from the two-dimensional numerical simulation of a plane mixing layer have been used to study the feasibility of tagging one side of the flow by a passive scalar and using the instantaneous concentration of the scalar to detect the typical coherent events in the flow. The study has shown that this technique works quite satisfactorily and yields results similar to those obtained by using the instantaneous vorticity as a detection criterion. The contribution from the coherent events to the time-averaged turbulent momentum and scalar transport has been estimated. It is found that this contribution is of the same order as the time-mean transport during most of the dynamical evolution of the coherent structure. However, it may attain very large values for short periods of time in the neighborhood of pairing. The increase is particularly spectacular in the case of the Reynolds shear stress. While the present findings obtained from a two-dimensional simulation seem to support earlier results obtained from actual experiments, it is desirable to conduct additional studies with three-dimensional simulations when they become available.
\end{abstract}

\section{INTRODUCTION}

Several techniques have been used in the past to educe and study organized structures in turbulent shear flows. These vary from simple level detection of the velocity signal to the use of various types of short-time averages of the flow properties. A relatively simple technique, which proved to be very successful in a recent study of the two-dimensional turbulent wake of a flat plate, consisted of using heat as a passive scalar to tag the flow. ${ }^{1}$ In this experiment, the free stream on one side of the wake was maintained at a uniform$l y$ higher temperature relative to the free stream on the other side. The resulting instantaneous temperature levels in the flow were found to provide a convenient and simple means of detecting the arrival of organized structures at the probe. In fact, with this technique, it was possible to isolate and study organized structures in the flow even at distances of the order of 250 momentum thicknesses downstream of the trailing edge using only single-point measurements. The results of this study are in good agreement with the recent findings of other researchers who have used more complex techniques for the eduction and analysis of the organized structures. ${ }^{2,3}$

The success of the heat-tagging technique in detecting organized large-scale structures in the flow depends on the existence of a strong correlation between these structures and the passive-scalar transport. This correlation, in turn, depends on the manner of introduction of the scalar, and appears to be the strongest when the scalar is introduced uniformly over one entire side of the wake (as opposed to introduction at the trailing edge, for example). The importance of the manner of introduction of the scalar is also apparent from the experiments of Cimbala, Nagib, and Roshko ${ }^{4}$ in the two-dimensional wake of a cylinder, which clearly showed that the flow field inferred from visualization of the passive scalar depends critically on the location of the point of introduction of the scalar contaminant.

The detailed implications of the scalar-tagging technique can be better understood if it is tested under conditions when the available flow information is not limited by singlepoint measurements. It was, therefore, decided that this technique be applied to study the large-scale organized structure of a numerically generated two-dimensional mixing layer, in which case information on the entire flow field is available at all instants of time. The present paper describes the results of such a numerical study.

\section{FLOW STUDIED}

The flow studied is a plane mixing layer, shown in Fig. 1. The database is produced by the numerical computations described in Sandham and Reynolds, ${ }^{5}$ using a random walk

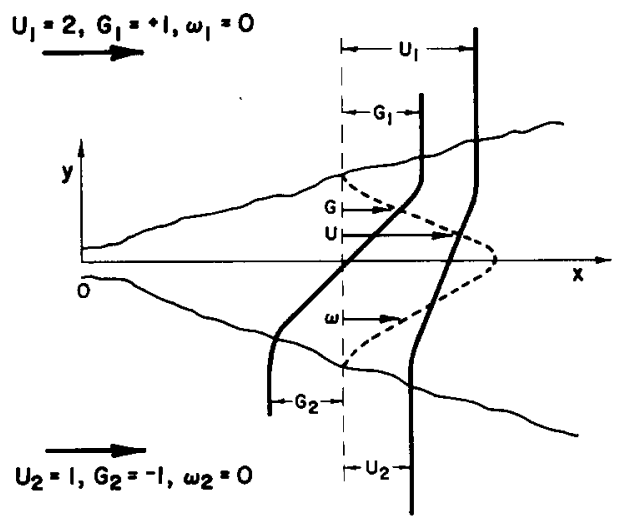

FIG. 1. The two-dimensional mixing layer. 
on the phase of the forcing eigenfunctions to simulate the natural mixing layer. The computations are made for the mixing layer between two streams with scalar concentrations $G_{1}=1$ ("high scalar" side) and $G_{2}=-1$ ("low scalar" side), and moving at velocities $U_{1}=2$ and $U_{2}=1$, respectively. The code is two dimensional, i.e., the two-dimensional instantaneous Navier-Stokes equations are solved. This is a limitation of the present study and is imposed by our current computational capabilities and resources. The computational domain extends from $x=0$ to $x=200$ initial vorticity thicknesses in the streamwise direction and $y=-\infty$ to $y=+\infty$ in the normal direction. The flow development within this domain is computed from the initial time $t=0$ to a time corresponding to 640 vorticity time units. Hyperbolic-tangent distributions are assumed for the mean velocity and scalar concentrations at $x=0$. Also, at this initial station, velocity perturbations of frequencies corresponding to the fundamental and two subharmonic instability modes of the mixing layer, but with a randomly walked phase, are introduced. At the downstream boundary, a convective-outflow condition (Lowery and Reynolds $^{6}$ ) is applied, which allows the structures to leave smoothly the computational domain. The numerical simulation corresponds to an inlet Reynolds number [based on the velocity difference $\left(U_{1}-U_{2}\right)$ and vorticity thickness] of 100 , and an outlet Reynolds number of approximately 1000 . A Schmidt number of 1 has been assumed for the scalar. The simulation was performed on a CRAY X-MP computer at the NASA Ames Research Center, and the post processing of the data was performed on a VAX 11/780 computer.

Figures 2(a) and 2(b) show typical snapshots of the simulated scalar and vorticity fields at $t=264$. These snapshots indicate the presence of single, as well as paired vortical structures, very similar to those observed in experiments. The simulated mixing layer has also been found to agree reasonably well with experimental measurements with respect to growth rate, and distributions of mean velocity, mean scalar concentration, and turbulent stresses. ${ }^{5}$ Hence, in spite of the rather low Reynolds number and two-dimensionality of the simulation, the numerical data can be considered to be adequate for the purpose of the present studies. In

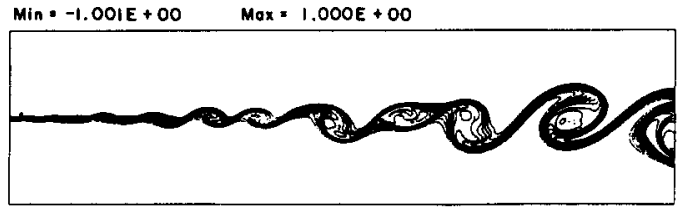

(a)

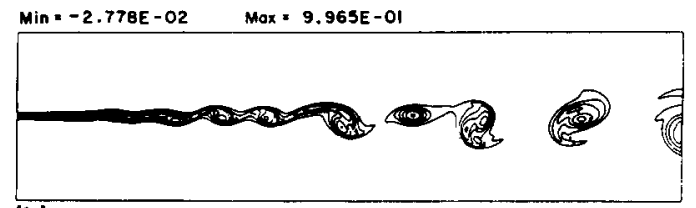

(b)

FIG. 2. Snapshots of the numerically simulated mixing layer at $t=264$. (a) Scalar contours. (b) Vorticity contours. Contours are drawn at equal intervals. Dashed lines indicate negative values. order to avoid the effects of the initial conditions, only data corresponding to $t>240$ have been used in the study. Likewise, data corresponding to two locations only, namely $x=78$ and $x=137$, have been used. These locations are sufficiently far away from the boundaries and hence are not directly influenced by the specified boundary conditions.

\section{EDUCTION PROCEDURE AND RESULTS}

It was decided that we study the organization in the flow in terms of coherent events in time rather than coherent structures in space. The study thus simulated the experimental situation in which fixed probes measure flow properties as a function of time. Furthermore, averages taken over the events have direct significance since they represent the contribution by the coherent activity to the "conventional" time-averaged properties. The procedure used for the eduction of the organized events in the flow was, in principle, similar to that used in the experiments described in Jovic and Ramaprian ${ }^{1}$ and can be understood from Fig. 3. Briefly, the method is as follows. First, a given $x$ location is selected for the study. The instantaneous velocity, vorticity, and scalar values at this station are each organized in the form of a time series for each $y$ location. A $y$ location in the "high scalar" side sufficiently away from the $y=0$ line is selected (arbitrarily to begin with). Based on the reasoning that a scalar value of less than 1 at this location indicates contamination with fluid from the "low scalar" side brought in by the large eddies, the scalar time series is scanned to detect the time instants when the scalar level crosses a prescribed threshold (say 0.95 ). The interval $t_{i}$ between two such crossings from 1 to 0.95 can be regarded as the interval of observation of a large "event," at the location of the detection point. This interval is a measure of the duration of the event. All such events are collected and a histogram of the intervals $t_{i}$ is generated. Also, the mean and standard deviation of these intervals are evaluated. The $y$ location and the threshold level are optimized so as to minimize the standard deviation of the intervals. (It was, however, verified that the overall conclusions did not critically depend on the precise choice of the location and threshold level.) The resulting normalized histogram is shown in Fig. 4 for the station $x=78$. The mean and normalized standard deviation of the intervals were found to be 10.3 and 0.315 , respectively. The figure also shows that the present histogram compares reasonably well
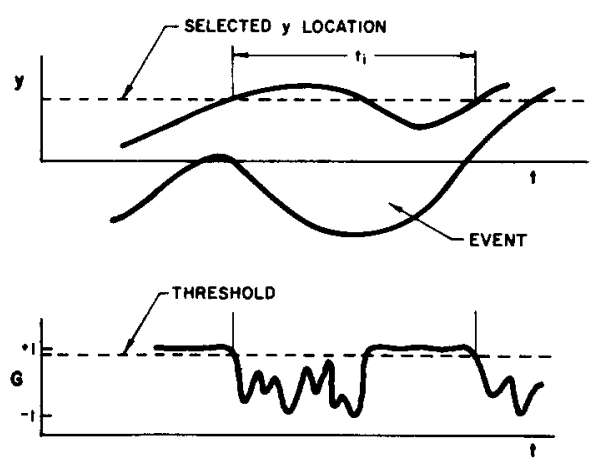

FIG. 3. An explanation of the eduction procedure. 


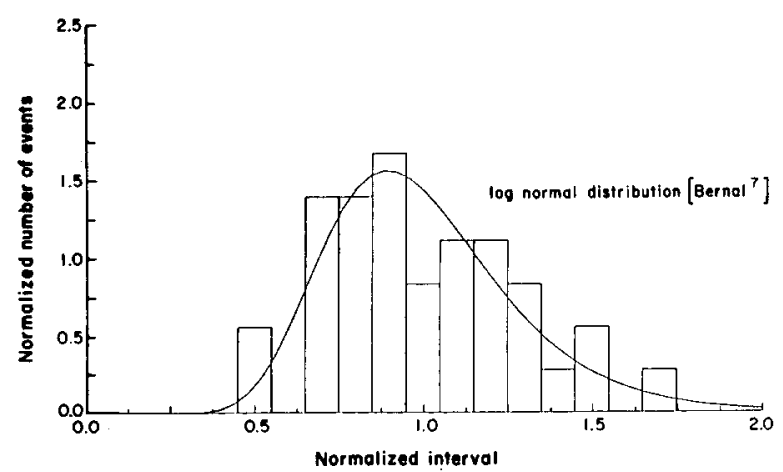

FIG. 4. A histogram of event intervals. Station $x=78, y=2.4$ scalar threshold $=0.95$. Mean duration $=10.3, \sigma=0.315$.

with the lognormal distribution predicted for a two-dimensional mixing layer by Bernal. ${ }^{7}$ The normalized standard deviation of 0.315 of the present data is comparable with the value of 0.276 obtained by Bernal. The agreement between the two indicates that the random-walk phase model used in the simulation algorithm yields a realistic description of the mixing layer.

The next step in the analysis consists of selecting one interval $t_{i}$ and obtaining at all $y$ locations at a fixed $x$ station ( $x=78$, say), ensemble-averaged values of the velocity components $U, V$, the vorticity $\omega$, and the scalar concentration $G$ at several instants during the event. For this purpose, the time coordinate $\tau$ is measured relative to the instant of detection of the event. The values of the properties, $U, V$, and $\omega$ at several instants preceding, during, and following the instant of detection are read from the data base for each event of interval $t_{i}$. These values are then ensemble averaged over all these events. From these ensemble averages, contours of these properties within the event are constructed. A typical contour plot of the ensemble-averaged scalar field associated with events of $t_{i}=9$ is shown in Fig. 5. These contours closely resemble the snapshots of individual realizations shown in Fig. 2(a), confirming that the procedure has successfully captured the typical events.

Contour plots were likewise obtained for other values of $t_{i}$ in the neighborhood of the mean value of 10.3. These were found to be very nearly identical (except for the stretching of the time scale) in the range $9 \leqslant t_{i} \leqslant 12$. It has been shown (Browand and Weidman ${ }^{8}$ ) that the large-scale vortical fea-

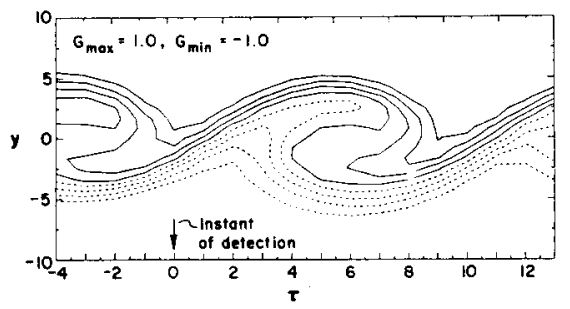

FIG. 5. Contours of ensemble-averaged scalar concentration, associated with events of interval $t_{i}=9$, at station $x=78 ; y=2.4$. Note that the contours extend from $\tau=-4$ (four time units preceding the detection of the event at this $y$ location) to $\tau=+13$ (four time units following the disappearance of the event at this $y$ location). ture of the mixing layer is in a state of continuous evolution from the instant of its generation to the instant when it pairs with a neighboring structure. The "events" corresponding to different intervals $t_{i}$ in the present study can be interpreted as realizations corresponding to different stages in this evolution process. The observed similarity of the contours over a range of $t_{i}$ values suggests that the dynamical evolution represented by these realizations can be treated, as a first approximation, as belonging to one characteristic phase in the overall evolution process. During this phase, there is no significant interaction between one structure and neighboring structure. This phase was found to occupy nearly $50 \%$ of the total time at the detection location. These realizations will be lumped together and referred to as typical events, even though they may represent simply different stages in the dynamical evolution of the vortical structures. A small number of realizations corresponding to values of $t_{i}$ toward the tail of the histogram in Fig. 4 were found to exhibit a structure completely different from that of the typical events. These realizations can be considered to represent a second distinct phase in the evolution of the vortical structures. This phase was found to correspond to pairing and was characterized by a strong interaction between adjacent structures. This phase was observed over a very small fraction of the total time at the detection locations studied. The realizations exhibiting this phase will therefore be referred to as atypical events.

The broad classification of the realizations into typical and atypical events is admittedly very coarse. However, a separate study by Sandham et al. ${ }^{9}$ of the scalar entrainment process in mixing layers provides some justification for this approach. In that study, the temporal evolution of a pair of vortical structures in the mixing layer was numerically simulated. The simulation (also two dimensional) covered the period from the inception to the pairing of the structures. The study showed that during this evolution, the rate of entrainment of scalar by the structures remained nearly constant over a substantial initial period, but rapidly increased in the neighborhood of pairing. The study also suggested that for a given Schmidt number of the scalar, the contrast between these two phases becomes sharper as the Reynolds number increases. It thus seems that a substantial amount of information about the coherent events can be obtained even from this broad classification of the evolution process into two phases. In any case, it is to be noted that it is possible, in principle, to use the scalar tagging technique to capture and average coherent events representing an arbitrarily large number of intermediate phases in the dynamical evolution, provided a large-enough database is available. Such a procedure requires extremely large computational effort and resources, and is outside the scope of the present study.

\section{ANALYSIS OF THE TYPICAL EVENTS}

As a final step in the analysis of the typical events, the contours for each $t_{i}$ in the range $9 \leqslant t_{i} \leqslant 12$ are normalized by the respective value of $t_{i}$. The normalized contours are then averaged over all these values of $t_{i}$, to obtain smoothed contours associated with the typical events. Figures 6(a) and 6(b) show typically the results for the normalized scalar concentration $G$ and the vorticity $\omega$, respectively, in the typi- 

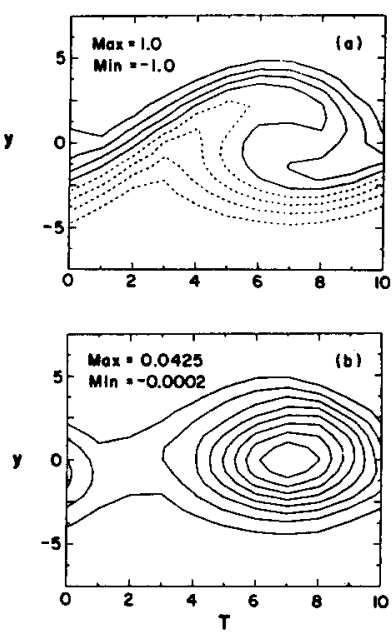

FIG. 6. Normalized contours of ensemble-averaged properties within a typical coherent event. $x=78$. (a) Scalar contours. (b) Vorticity contours.

cal event. The contours are drawn for equal intervals. Since at this station the typical coherent events appear almost periodically (as seen from Fig. 5), only one event is shown in Figs. 6(a) and 6(b). Note that the time axis in these figures is scaled such that the overall duration of the event is equal to 10 units, with $T=0$ and $T=10$ representing the beginning and end of the event, respectively. These contours are analogous to those that would be obtained in an experiment from the instantaneous outputs of a multitude of probes located across the mixing layer at the given $x$ station, as a coherent event passes by the station. The figures clearly show that the scalar-conditioning technique has been successful in educing the typical coherent events in the flow. A secondary observation that can be made from the figures is that while details within the event may differ, there is an overall correspondence between the scalar and vorticity fields associated with the coherent event. This correspondence explains the success of the scalar-conditioning technique in educing the coherent (vortical) events in the flow. It is to be noted that while vorticity is expected to behave like a scalar in a twodimensional simulation, the boundary conditions for the two are quite different in the present case. The distributions of the scalar concentration and vorticity can therefore be expected to be different, as indeed they are. The two distributions resemble each other only with respect to the overall topology such as the shape and size of the outer contours and not with respect to the details of distribution. For example, the scalar contours can be used to distinguish between the fluid from the two sides of the mixing layer, whereas the vorticity contours do not provide this information. Also, it is very clearly seen that the scalar gradients are very strong within the braid but there is hardly any spanwise vorticity carried by the braid. It is, however, important to note that in this case, the Schmidt number of the passive scalar is of order 1 and that the scalar is "introduced" at all $x$ locations instead of at one upstream location.

The availability of information on the instantaneous flow field provides an opportunity to study the detailed structure of these typical coherent events and their contribution to the overall turbulent transport. One can estimate, for example, the contribution from the coherent events to the conventionally defined turbulent shear stress $\overline{u^{\prime} v^{\prime}}$ and the turbulent scalar transport $\overline{u^{\prime} g^{\prime}}$ and $\overline{v^{\prime} g^{\prime}}$ in the streamwise and normal directions, respectively. During a coherent event, the instantaneous velocity fluctuations $u^{\prime}$ and $v^{\prime}$ with respect to the long-time-average velocity $\bar{U}$ and $\bar{V}$, and the instantaneous scalar concentration fluctuation $g^{\prime}$ with respect to the long-time-averaged scalar concentration $\bar{G}$ can, respectively, be written as

$$
\begin{aligned}
& u^{\prime}=\langle U\rangle+u_{\text {sup }}, \\
& v^{\prime}=\langle V\rangle+v_{\text {sup }},
\end{aligned}
$$

and

$$
g^{\prime}=\langle G\rangle+g_{\text {sup }},
$$

where the \langle\rangle denotes ensemble-averaged values with respect to the corresponding long-time-average value and the subscript "sup" denotes the random departure of the instantaneous value from the ensemble average. The latter can be regarded as "turbulence" superposed on the deterministic fluctuations. The instantaneous products $u^{\prime} v^{\prime}, u^{\prime} g^{\prime}$, and $v^{\prime} g^{\prime}$ can then be ensemble averaged for each nondimensional time $\tau / t_{i}$ within the typical coherent event and subsequently averaged over the entire duration of the event to obtain

$$
\begin{aligned}
& \overline{\left\langle u^{\prime} v^{\prime}\right\rangle}=\overline{\langle U\rangle\langle V\rangle}+\overline{\left\langle u_{\text {sup }} v_{\text {sup }}\right\rangle}, \\
& \overline{\left\langle u^{\prime} g^{\prime}\right\rangle}=\overline{\langle U\rangle\langle G\rangle}+\overline{\left\langle u_{\text {sup }} g_{\text {sup }}\right\rangle}, \\
& \overline{\left\langle v^{\prime} g^{\prime}\right\rangle}=\overline{\langle V\rangle\langle G\rangle}+\overline{\left\langle v_{\text {sup }} g_{\text {sup }}\right\rangle} .
\end{aligned}
$$

The terms on the left-hand side of the above equations represent the conditionally averaged contribution from the typical coherent events to the time-mean transport. The variations of these terms across the mixing layer can be regarded as the average distribution of the transport terms across the mixing layer during the occurrence of a typical coherent event. The terms on the right-hand side represent the contributions, respectively, from the organized (deterministic) and random (superposed) parts of the fluctuations associated with this typical event. The decomposition and analysis represented by Eqs. (1)-(6) are similar to those described in Jovic and Ramaprian. ${ }^{1}$ Typical results for the station $x=78$ are shown in Figs. 7 (a) -7 (c) for the shear stress, and the scalar transport in the longitudinal and normal directions, respectively. The distributions of the corresponding timemean terms are also shown in these figures. First, it is seen that the superposed turbulence (which can be regarded as representing the combination of any random variations associated with the coherent structures, effect of nonsimilar dynamic evolution, and any jitter associated with the process of ensemble averaging) is as large as the organized component in the case of the shear stress but is less significant in the case of the scalar flux. However, at the downstream location $x=137$, the level of the superposed shear stress also was found to have a relatively small magnitude. Second, the conditionally averaged Reynolds stress $\left\langle\overline{u^{\prime} v^{\prime}}\right\rangle$, given by the sum of the above two components in Fig. 7 (a), is seen to be about $60 \%$ of the time-mean value. At the downstream station, the conditional average was found to be approximately equal in magnitude to the time-mean value. Figures $7(\mathrm{~b})$ and $7(\mathrm{c})$ show similar trends for the scalar transport in the streamwise and normal directions. It is thus concluded that the 


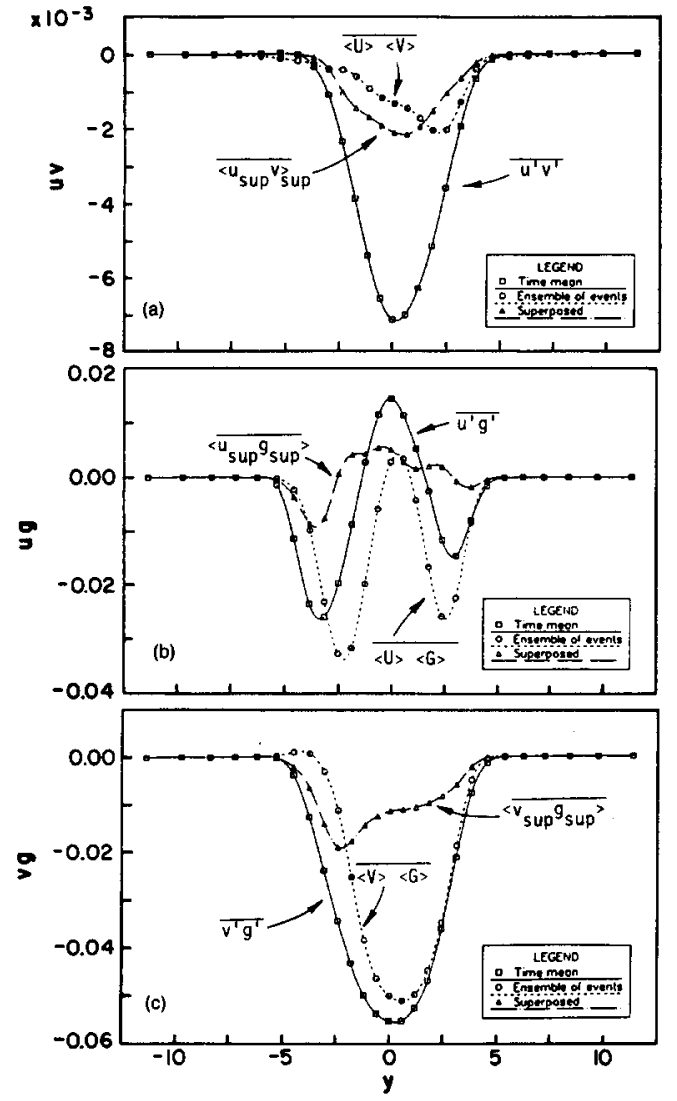

FIG. 7. Contribution from the typical coherent event to turbulent transport. Station $x=78$ (a) Shear stress, (b) streamwise scalar transport, and (c) cross-stream scalar transport.

typical coherent events carry shear stress and heat flux of approximately the same magnitude as the corresponding time-mean values. Hence these typical coherent events can be considered to be significant events in the flow (but not necessarily the most significant) from the point of view of turbulent transport. The result, however, differs from the experimental results for the two-dimensional wake, ${ }^{1}$ which indicated that the shear stress associated with the coherent motion was two to three times larger than the time-mean value. This difference in the results can be attributed to the fundamental differences between the dynamics of the mixing layer and the far wake (such as the absence of coherent events over a significant fraction of the time, and the absence of pairing in the far wake), though other experimental features, such as three-dimensionality and contamination with atypical events during eduction, may have contributed somewhat to the observed differences.

\section{RESULTS FOR THE ATYPICAL EVENTS}

The growth of the mixing layer is characterized by pairing of the coherent structures. Experiments indicate that these pairings occur over a wide range of streamwise $(x)$ locations, with the average distance between successive locations increasing linearly with the width of the mixing layer. However, it is likely that at any $x$ location, a pairing event can be observed if one waits for a sufficiently long time. Because of the restricted space-time domain of the present numerical simulation, it was not possible to find a sufficient

number of such pairing events that were required for the estimation of the ensemble-averaged properties. Furthermore, large changes occur during the pairing process. For example, Lowery and Reynolds ${ }^{6}$ have shown that the shear stress becomes negative during the time that one structure is directly above the other (i.e., vertically aligned) and recovers as the top structure rolls over the lower one. Hence meaningful ensemble averages can be estimated only by collecting many pairing events at the same stage of evolution. This process calls for an even longer time record. In the absence of such a long record, the best that can be done is to study some individual realizations belonging to some significant stage of evolution during the pairing process. The results of such a study can then be used to obtain some quantitative measure of the contribution to the overall turbulent transport from the pairing event as a whole. It should be noted that this simplified approach is necessitated not by the limitation of the education technique but by the available numerical database. The passive scalar tagging technique was as effective in educing these atypical events as it was in the case of the typical events.

In the present study, the individual realizations chosen corresponded to the instant when one structure was approximately at $-45^{\circ}$, with respect to the neighboring structure during the pairing process. It appears that the contribution to the overall turbulent transport is maximum at this instant. The results for one such realization detected at $x=137$ are discussed here. These results can be considered to be representative of this phase in the evolution of the coherent events.

Figures 8(a) and 8(b) show the scalar concentration and vorticity contours for the above realization. Once again, overall topological correspondence can be observed between the scalar and vorticity fields. However, the scalar gradients in the interior of the event are less strong than those of vorticity, which shows a nearly uniform variation from the center to the outer edge of the event. Figures 9 (a) -9 (c) show the cross-stream distribution of the transport terms for momentum and scalar, averaged over the event. The same nomenclature as was used in Figs. 7(a)-7(c) has been retained even though the "ensemble average" has been obtained over only one realization and hence the superposed component is zero. It is seen that the maximum conditionally averaged shear stress associated with this atypical event is about eight times larger than the time-mean maximum shear stress in
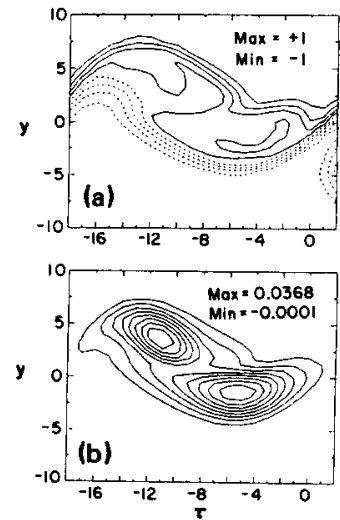

FIG. 8. Contours of properties within a pairing event. Station $x=137$. (a) Scalar concentration; (b) vorticity. 

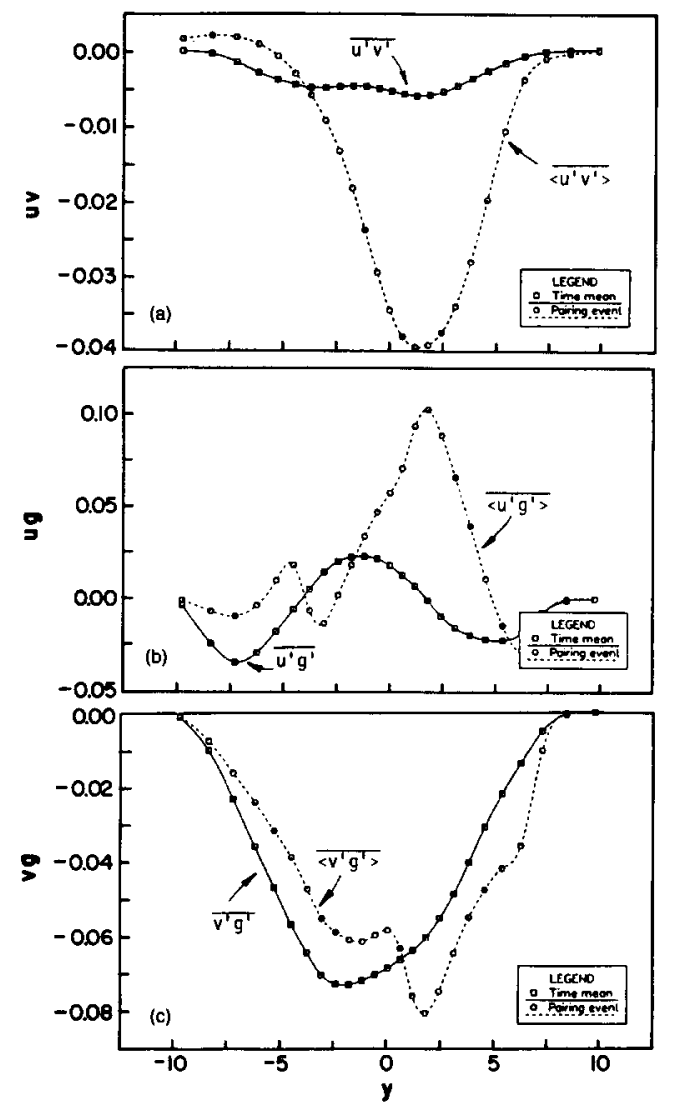

FIG. 9. Contribution from the pairing event to turbulent transport. Station $x=137$. (a) Shear stress; (b) streamwise scalar transport; and (c) crossstream scalar transport.

the mixing layer. On the other hand, the contribution from this event to the cross-stream scalar transport is of the same order as the time-mean value. The contribution in the case of the longitudinal transport is somewhat larger but is by no means dominant, especially if the results are averaged over the cross section of the mixing layer. Comparing these results with those for the typical coherent event, one can conclude that pairing is responsible for bringing about a significant amount of turbulent transport. The contribution to the transport of momentum is indeed spectacular during pairing, while the contribution to scalar transport is only moderate. The present results with respect to the contribution from the pairing process to the Reynolds shear stress production are in agreement with the experimental observations of Browand and Weidman. ${ }^{8}$ The results shown in Figs. 9(a)9(c) may be modified slightly if ensemble averages are obtained over a large number of realizations. However, the events are so strongly coherent that it is unlikely that a major departure from this trend would emerge from the study of a large number of realizations.

\section{COMPARISON OF SCALAR CONDITIONING WITH VORTICITY CONDITIONING}

Finally, it is instructive to compare the present eduction technique based on scalar-level conditioning with another established technique. Comparisons of the present results were made with those obtained using the vorticity level for the eduction of the coherent events. Vorticity is often regarded as the best criterion for the identification of the coherent structures, ${ }^{3}$ although the instantaneous vorticity vector is one of the most difficult quantities to monitor in an experiment. In the present two-dimensional simulation, however, there is only one nonzero component of the vorticity vector and instantaneous values of this component were readily available. The eduction procedure used was analogous to that used with the scalar. Thus a suitable $y$ location near the edge of the mixing layer and a vorticity threshold level are selected by trial and error. Crossings of this level by the vorticity values in the increasing direction from zero are assumed to be associated with the passage of the coherent (vortical) events. An optimized histogram of the intervals and the ensemble averages of the properties are then obtained as in the case of scalar conditioning. Figure 10 shows the histogram of the intervals of the coherent events, at the detection point. This can be compared with Fig. 4. It is seen that while there are some differences in detail, the two histograms are quite similar. The mean interval of the events is nearly the same in the two cases. So also are the intervals of the most frequent events. In fact, the contours of ensembleaveraged scalar concentration and vorticity were found to be indistinguishable from those shown in Figs. 6(a) and 6(b) and are therefore not presented separately. The contours of ensemble-averaged shear stress $\langle U\rangle\langle V\rangle$ (corresponding to the organized motion) obtained with the two techniques are compared in Figs. 11(a) and 11(b). It is seen that there are only very small differences between the two results. For example, there is only a $4 \%$ difference in the maximum values (and also minimum values) for the two cases. A larger difference (about $25 \%$ ) is observed in the estimated peak value of the conditionally averaged shear stress associated with the typical coherent events (Fig. 12). Even in this case, the discrepancy between the two techniques becomes negligibly small, if the results are averaged over the cross section. The differences between the results obtained from the two techniques are thus well within the uncertainties to be expected in a study such as this, especially when a small number of realizations are used to obtain the ensemble averages.

The present tests using two-dimensional simulations do not by themselves provide conclusive evidence of the applicability of the scalar-tagging technique in instantaneously three-dimensional flow fields. However, the present findings taken together with the successful results obtained in an actual plane wake, ${ }^{1}$ seem to indicate that the technique, if properly used, is capable of detecting coherent structures in these flows as reliably as the more difficult vorticity-condi-

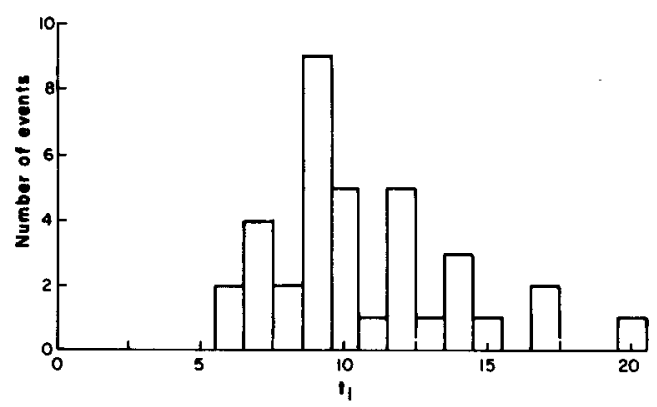

FIG. 10. A histogram of interval durations for a typical coherent event, obtained from vorticity conditioning. Station $x=78, y=2.4$, vorticity threshold $=0.04$. Mean interval $=10.6, \sigma=0.31$. 


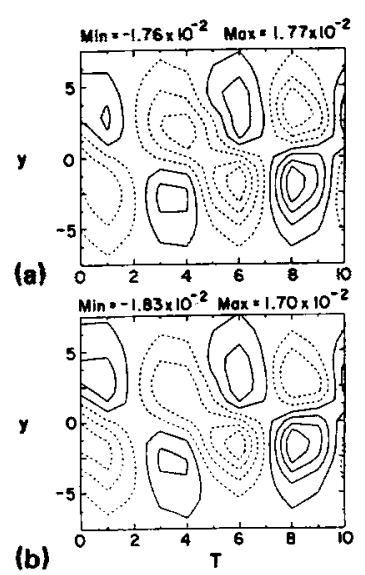

FIG. 11. Contours of the ensemble-averaged shear stress $\langle U\rangle\langle V\rangle$ in the typical coherent event. Station $x=78$. (a) From scalar conditioning; (b) from vorticity conditioning.

tioning technique. In fact, the three-dimensionality of the instantaneous flow field in such cases introduces conceptual as well as measurement difficulties in using the instantaneous vorticity vector as a criterion for detection. Scalar measurements do not pose such difficulties.

\section{DISCUSSION}

The present study has shown that by tagging the free stream on one side it is possible to educe the coherent structures in the mixing layer. In fact, eduction based on scalar conditioning was found to capture more or less the same structures as those captured by a common alternate technique such as vorticity conditioning, even though there are differences in detail between the scalar and vorticity fields within the structure. The reason for this is the fact that under the present tagging conditions, large-scale organization in the vorticity field leads to a corresponding organization in the scalar field, which is relatively easy to detect.

It is important to note that the manner in which the scalar tagging is implemented in the present case allows one to distinguish between the two sides of the mixing layer. Thus the presence of low scalar concentrations on the "high" side is primarily the result of large-scale activity in the flow. This strong correlation is the reason why the coherent events can be detected using a simple level criterion, even in the presence of noise in the experiments. Under the present scalar-tagging conditions, the large-scale scalar field is essentially driven by the scalar boundary conditions rather than by diffusion or three-dimensional effects. Therefore it is a reasonable expectation that the technique will work, even when the Schmidt number of the scalar is not unity and the instantaneous flow field is three dimensional. In fact, the effect of Schmidt number becomes insignificant at very high Peclet numbers (the product of Reynolds number and Schmidt number).

Another merit of the present method for the study of plane mixing layers and wakes is that since the entire free stream on one side is tagged, the signal-to-noise ratio is uniformly high at all streamwise locations. On the other hand, with vorticity tagging (or any type of tagging of the initial vortical fluid), the signal-to-noise ratio would decrease with

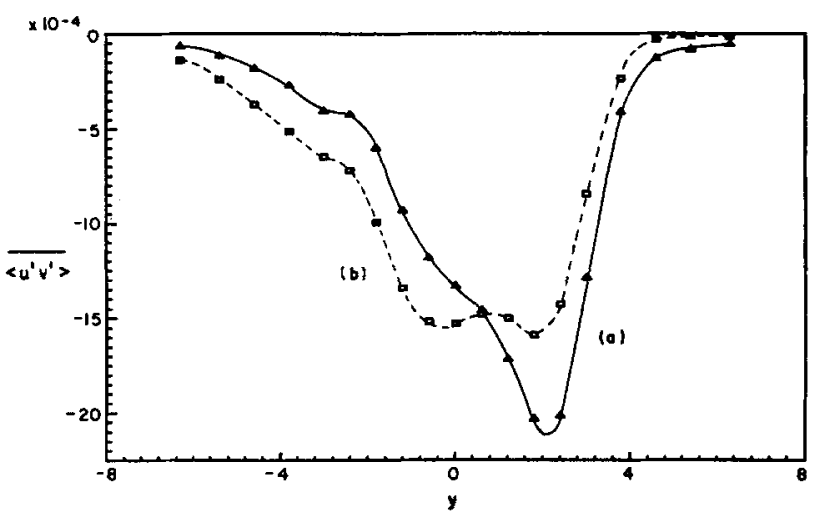

FIG. 12. Distribution of the conditionally averaged shear stress across the mixing layer. (a) From scalar conditioning; (b) from vorticity conditioning.

downstream distance, making it very difficult to use the signal for the identification of the coherent events in the intermediate-to-far field. Note, however, that instantaneous scalar distributions are known to have sharper edges than those of velocity distributions and hence can be more effectively used for the detection of the passage of large-scale structures. Hence even heat tagging of the upstream fuid has been successfully used in the past for the study of large-scale structures in jets and wakes (e.g., Antonia et al. ${ }^{10}$ and LaRue ${ }^{11}$ ).

It is also essential to note in this connection that while scalar tagging can be used in a relatively simple manner to detect the coherent events, obtaining ensemble-averaged properties from a collection of such events poses exactly the same problems as those encountered with other techniques. These include problems such as scaling and alignment of the individual events, which require careful consideration, especially in the presence of three-dimensionality.

\section{CONCLUSIONS}

The present study has demonstrated that, if properly used (i.e., by tagging one of the free streams), passive-scalar tagging can be a simple and effective technique for the eduction and analysis of coherent structures in plane turbulent free shear flows. The study of the ensemble-averaged properties associated with the coherent events has shown that in a plane mixing layer, the typical coherent events (representing the situation wherein the interaction between neighboring vortical structures is not very energetic) carry a significant amount of "turbulence" and can therefore affect the overall transport of momentum and scalar in a significant manner. The rate of transport reaches very large values during the atypical events (representing pairing between neighboring vortical structures). This is particularly spectacular in the case of the rate of transport of momentum, which reaches several times its time-mean value, during a pairing event. The scalar concentration gradients in the interior of the structure after a pairing process are much smaller than the velocity gradients, resulting in smaller scalar transport compared to momentum transport. 
The present conclusions are based on the study of the two-dimensional simulation of a mixing layer. While there is some good evidence to suggest that these conclusions would be valid, even in the presence of three-dimensionality of the instantaneous flow field, it is still desirable to confirm them by studying three-dimensional numerical simulations.

\section{ACKNOWLEDGMENTS}

This work was carried out as a part of the 1988 Summer Research Program of the NASA-Stanford Center for Turbulence Research.

The support of this work by CTR is gratefully acknowledged.
${ }^{1}$ S. Jovic and B. R. Ramaprian, Phys. Fluids A 1, 331 (1989).

${ }^{2}$ L. W. B. Browne, R. A. Antonia, and D. K. Bisset, Phys. Fluids 29, 3612 (1986).

${ }^{3}$ A. K. M. F. Hussain and M. J. Hayakawa, J. Fluid Mech. 108, 193 (1987).

${ }^{4}$ J. M. Cimbala, H. M. Nagib, and A. Roshko, J. Fluid Mech. 190, 265 (1988).

${ }^{5}$ N. D. Sandham and W. C. Reynolds, in Turbulent Shear Flows 6 (Springer, New York, 1989), p. 441.

${ }^{6}$ P. S. Lowery and W. C. Reynolds, Report No. TF 26, Stanford University, 1986.

${ }^{7}$ L. P. Bernal, Phys. Fluids 31, 2533 (1988).

${ }^{8}$ F. K. Browand and P. D. Weidman, J. Fluid Mech. 76, 127 (1976).

${ }^{9}$ N. D. Sandham, M. G. Mungal, J. E. Broadwell, and W. C. Reynolds, Proceedings of the 1988 Summer Program, Report No. CTR-S88, NASAStanford Center for Turbulence Research, Stanford University, p. 69, 1988.

${ }^{10}$ R. A. Antonia, A. Prabhu, and S. E. Stephenson, J. Fluid Mech. 72, 455 (1975).

${ }^{11}$ J. C. LaRue, Phys. Fluids 8, 1513 (1974). 